\title{
Variable neuroprotective role of Filipendula ulmaria extract in rat hippocampus
}

\author{
Natalija Arsenijevic ${ }^{1, \dagger}$, Dragica Selakovic ${ }^{2, \dagger}$, , Jelena S. Katanic Stankovic ${ }^{3}$, Vladimir Mihailovic ${ }^{4}$, Slobodanka Mitrovic ${ }^{5}$, \\ Jovana Milenkovic ${ }^{1}$, Pavle Milanovic ${ }^{1}$, Miroslav Vasovic ${ }^{1}$, Aleksandra Nikezic ${ }^{6}$, Olivera Milosevic-Djordjevic ${ }^{6,7}$, \\ Marko Zivanovic ${ }^{3,8}$, Nenad Filipovic ${ }^{8,9}$, Vladimir Jakovljevic ${ }^{2,10}$, Nemanja Jovicic ${ }^{11}$, Grozden Rosic ${ }^{2, *}$ \\ ${ }^{1}$ Department of Dentistry, Faculty of Medical Sciences, University of Kragujevac, 34000 Kragujevac, Serbia \\ ${ }^{2}$ Department of Physiology, Faculty of Medical Sciences, University of Kragujevac, 34000 Kragujevac, Serbia \\ ${ }^{3}$ Department of Science, Institute for Information Technologies Kragujevac, University of Kragujevac, 34000 Kragujevac, Serbia \\ ${ }^{4}$ Department of Chemistry, Faculty of Science, University of Kragujevac, 34000 Kragujevac, Serbia \\ ${ }^{5}$ Department of Pathology, Faculty of Medical Sciences, University of Kragujevac, 34000 Kragujevac, Serbia \\ ${ }^{6}$ Department for Biology and Ecology, Faculty of Science, University of Kragujevac, 34000 Kragujevac, Serbia \\ ${ }^{7}$ Department of Cenetics, Faculty of Medical Sciences, University of Kragujevac, 34000 Kragujevac, Serbia \\ ${ }^{8}$ BiolRC, Bioengineering RED Center, 34000 Kragujevac, Serbia \\ ${ }^{9}$ Faculty of Engineering, University of Kragujevac, 34000 Kragujevac, Serbia \\ ${ }^{10}$ Department of Human Pathology, First Moscow State Medical University IM Sechenov, 119992 Moscow, Russia \\ ${ }^{11}$ Department of Histology and Embryology, Faculty of Medical Sciences, University of Kragujevac, 34000 Kragujevac, Serbia \\ "Correspondence: grosic@medf.kg.ac.rs (Gvozden Rosic) \\ ${ }^{\dagger}$ These authors contributed equally. \\ DOI:10.31083/j.jin2004089 \\ This is an open access article under the CC BY 4.0 license (https://creativecommons.org/licenses/by/4.0/). \\ Submitted: 2 July 2021 Revised: 19 August 2021 Accepted: 30 August 2021 Published: 30 December 2021
}

We evaluated the influence of an antioxidant-rich extract of Filipendula ulmaria L. on anxiety levels induced by nano-sized particles of different calcium phosphates. Rats in experimental groups were administered with either nano-sized hydroxyapatite, tricalcium phosphate, or amorphous calcium phosphate in the presence of Filipendula ulmaria extract. Appropriate behavioral tests were performed to assess anxiety levels, while oxidative status and apoptosis parameters were determined in the hippocampus samples. The applied calcium phosphates increased oxidative stress markers in hippocampal tissue, accompanied by an enhanced pro-apoptotic mechanism. Moreover, the hippocampal immunoreactivity for brainderived neurotrophic factor and CABAergic-A receptors was significantly lower following calcium phosphate nanoparticles intake. The observed functional and morphological alterations in the rat hippocampus occurred simultaneously with the anxiogenic response estimated in behavioral testing. The neuroprotective effect of Filipendula ulmaria was markedly manifested by the attenuation of oxidative damage induced by amorphous calcium phosphate and enhanced anti-apoptotic action in the rat hippocampus. The increased hippocampal immunoreactivity for brain-derived neurotrophic factor, CABAergic-A receptors and significant anxiolytic-like effects of Filipendula ulmaria may suggest a beneficial role of antioxidant supplementation in preventing anxiogenic response to nano-sized calcium phosphates.

Keywords

Calcium phosphates nanoparticles; Filipendula ulmaria; Behavior; Anxiety; Apoptosis; Oxidative stress

\section{Introduction}

The results obtained in studies performed on cell culture indicate that cell damage was fundamentally caused by calcium phosphate nanoparticle impact on oxidative mechanisms with consequent apoptosis [1]. This has been confirmed by Xu et al. [2] that inhibition of osteoblast activity by hydroxyapatite (HA) nanoparticles was accompanied by apoptosis in vitro through the mitochondrial mechanisms they suggest. The extent of apoptosis was determined by the concentration and size of the HA nanoparticles. The association between cell apoptosis and the size of HA particles was also confirmed by Shi et al. [3] with osteoblast-like cells, while Liu et al. [4] and co-workers concluded that differences in the $\mathrm{Ca} / \mathrm{P}$ ratio of different $\mathrm{CaP}$ phases were among the key factors that determined the level of osteoblast apoptosis that should be considered, besides the particular size, in selecting the type CaPs used for various therapeutic applications. It is usually claimed that oxidative damage may be a substantial part of the pathophysiological mechanism that underlines the numerous toxicities induced by $\mathrm{CaP}$ compounds.

Meadowsweet, Filipendula ulmaria (L.) Maxim. - FU is a perennial herb that belongs to the Rosaceae family. Traditionally, the flowers of this plant are used to treat colds and mild rheumatic, renal, and gastric dysfunctions [5]. It is assumed that the aerial parts of FU have numerous medicinal effects: diuretics, antiseptics, antirheumatics, and antacids. 
Extensive research has shown that FU exhibits strong antioxidant, antimicrobial, cytotoxic, and anti-inflammatory properties [6-8].

Following the recent trend of administering natural products as antioxidant supplementation in the treatment of various disorders accompanied with oxidative damage, that include confirmation of their neuroprotective role in both preand clinical studies [9], there is a growing need for extensive use of calcium phosphates with a bioactive effect due to the confirmed toxic effects in various tissues caused by the use of composites containing nano-CaPs, as well as the fact that the neurotoxicity (including behavioral manifestations).

FU extract (aerial parts of the plant) was chosen in our earlier work [10], exploring the possibility of balancing the redox status altered by different nano-sized $\mathrm{CaP}$ compounds. Our previous investigations have confirmed that both prodepressant effect and cognitive decline were also accompanied by changes in the prefrontal cortex [10]. Here, the intention was to evaluate the potential influence of predefined protocols [10] on other mood disorders, such as the impact on anxiety state level and the different brain structures (hippocampus) responsible for mood regulation. Furthermore, we define morphofunctional alterations in the hippocampus, the region crucially involved in anxiety level regulation, following the chronic intake of nano-sized CaPs with and/or without antioxidant supplementation by FU extract.

\section{Materials and methods}

\subsection{Pretreatment protocols}

The MMA, Serbia, provided Nine-week-old male Wistar albino rats (weighting 180-220 g). Rats were housed in plexiglass cages (three rats in each), maintaining the controlled physiological environment (constant temperature $23 \pm 1{ }^{\circ} \mathrm{C}$ and humidity $50 \pm 5 \%$ ) with equal light and dark phases. The animals were allowed unrestricted food and water intake during the pretreatment protocols.

Rats were randomly assigned into seven groups (six animals per group). Control group; HA group, that received per os hydroxyapatite (17.8 mg/kg b.w.) for 30 days; HA + FU group, received per os hydroxyapatite $(17.8 \mathrm{mg} / \mathrm{kg}$ b.w. $)$ and FU extract (100 mg/kg b.w.) for 30 days; tricalcium phosphate (TCP) group, received per os tricalcium phosphate hydrate $(11 \mathrm{mg} / \mathrm{kg}$ b.w.) for 30 days; TCP + FU group, received per os tricalcium phosphate hydrate $(11 \mathrm{mg} / \mathrm{kg}$ b.w.) and FU extract (100 mg/kg b.w.) for 30 days; amorphous calcium phosphate (ACP) group, received per os amorphous calcium phosphate ( $9.65 \mathrm{mg} / \mathrm{kg}$ b.w.) for 30 days; and ACP + FU group received per os amorphous calcium phosphate $(9.65$ $\mathrm{mg} / \mathrm{kg}$ b.w.) and FU extract (100 mg/kg b.w.) for 30 days.

The characteristics of nano-sized CaPs, purchased from Sigma-Aldrich (Germany), matched those described previously in our work [10]. The Filipendula ulmaria extract preparation and content determination were performed as described in our previous work [6]. Doses of mineral components were equimolar to allow the comparison of individual
CaP effects. The applied doses met the lowest HA dose that induced toxic effects in vivo experiments with nanoparticles [11] and in vitro experiments, which evaluated the concentrations of daily CaPs released from dental composites [12]. To mimic the original route, all CaPs were applied orally. According to our previous results, the plant extract dose was chosen [7], where FU induced significant biological effects. The final concentrations of all estimated compounds were determined following the procedure described in our previous work [10].

The pretreatment and experimental procedures were performed following the European Directive for the Welfare of Laboratory Animals Directive 2010/63/EU, and the GLP principles, as well as the ARRIVE guidelines, following the approval by the Ethical Committee of the Faculty of Medical Sciences, University of Kragujevac, Serbia.

\subsection{Anxiety level estimation}

On the day following the end of the pretreatment protocols, rats were accommodated in the testing area for onehour (around $8 \mathrm{AM}$ ). Anxiety level estimation was performed in an open field (OF) and elevated plus maze (EPM) tests. The spontaneous activity in OF maze, followed by EPM, was recorded for five minutes. During 15 minutes between each trial, the mazes were cleaned with water and ethanol (70\%) to remove any potentially interfering scents that might affect the test. Video recordings were interpreted with Ethovision software XT 13.0.1220 (Noldus Information Technology, the Netherlands).

OF testing was conducted according to a previously defined algorithm in our lab [8]. The following parameters were calculated: the cumulative duration in the center zone CDCZ, in seconds, the frequency to the central zone - FCZ, the total distance moved - TDM, in centimeters, the percentage of time moving - \%TM, and the wall, free and total number of rearings.

EPM testing was also implemented according to an established standard procedure [13]. The anxiety indicators analyzed from the EPM test were: the cumulative duration in open arms - CDOA, in seconds, the frequency to open arms FOA, the total distance moved, in $\mathrm{cm}$, the percentage of time moving, the number of walls and free rearings, the number of protected and unprotected head-dippings, and the number of total exploratory activity (TEA) episodes.

\subsection{Tissue sample collection and analysis}

After the finalization of behavioral estimation, rats were anesthetized by the combination of ketamine and xylazine (10 and $5 \mathrm{mg} / \mathrm{kg}$, respectively, i.p.) and decapitated. The intact brain hemispheres were promptly and carefully separated and sagittally divided, one for immunohistochemistry and hippocampal tissue dissection (divided for RT PCR and oxidative stress marker analysis). 
2.3.1 Quantification of oxidative stress indicators in the hippocampus

According to a previously described procedure, the homogenization of hippocampal tissue and sample preparation (centrifugation at $4000 \mathrm{rpm}$ for $15 \mathrm{~min}$ at $4{ }^{\circ} \mathrm{C}$ in $\mathrm{PBS}, \mathrm{pH}$ 7.4) was performed [14]. Supernatants were distinct for further analyses using the materials presented in Supplementary file 1. All spectrophotometric analyses were performed using a UV-Vis double beam spectrophotometer (model Halo DB-20S, with a temperature controller, Dynamica $\mathrm{GmbH}$, Dietikon, Switzerland). The evaluation of protein content in the hippocampal samples followed Lowry's method [15], while BSA was used as the standard. The estimation of oxidative status in hippocampal tissue was analyzed according to the following indicators: index of lipid peroxidation (expressed as thiobarbituric acid reactive substances - TBARS), the activity of antioxidant enzymes (superoxide dismutase SOD and catalase - CAT), and non-enzymatic defense, expressed as the levels of reduced glutathione (GSH). The index of lipid peroxidation in hippocampal samples was assessed as the levels of TBARS and presented as nmol MDA/mg protein [16]. SOD activity was quantified spectrophotometrically [17], following the monitoring of the inhibition of (adrenalin) transformation to adrenochrome. The rate of hydrogen peroxide degradation was used to determine CAT activity determination [18]. The antioxidant enzymes activities were presented as $\mathrm{U} / \mathrm{mg}$ protein. The GSH level was quantified as previously described [19] and presented in mg GSH/g protein.

\subsubsection{Isolation of hippocampal RNA and RT-PCR analysis}

Extraction of total RNA from hippocampal samples followed the manufacturer's instructions and was performed using PureZOL reagent (Bio-Rad, USA). iScript Reverse Transcription Mastermix (Bio-Rad, USA) was used for reverse transcription. Quantitative RT-PCR was obtained with SsoAdvanced Universal SYBR Green Supermix (Bio-Rad, USA). mRNA-specific primers for Bax, Bcl-2, and $\beta$-actin (housekeeping gene), presented in Supplementary Table 1, were used, and quantitative RT-PCR reactions were performed in the Applied Biosystems 7500 (Applied Biosystems, USA). The relative gene expression was quantified according to Livak and Schmittgen [20].

\subsubsection{IHC analysis of hippocampal tissue}

A randomly-selected hemisphere for each rat was fixated in $4 \%$ formaldehyde solution in PBS and paraffinembedded. Five $\mu \mathrm{m}$ thick coronal brain sections were dewaxed, rehydrated, and antigen retrieval was done using citrate buffer ( $\mathrm{pH}$ 6.0) in a microwave for 23 minutes. According to manufacturer instructions, staining was visualized using an EXPOSE Rabbit-specific HRP/DAB detection IHC Kit (ab80437, Abcam, UK), and sections were counterstained with Mayer's hematoxylin. The slices were incubated with Anti-GABA A Receptor alpha 2/GABRA2 antibody (ab216973, dilution 1:250) and Recombinant Anti-
BDNF antibody [EPR1292] (ab108319, dilution 1:500) for 12 hours overnight at room temperature in a humidity chamber. Sections were covered with DPX mounting medium (Sigma, USA). The obtained hippocampal sections were photomicrographed by light microscope fitted with a digital camera (Olympus BX51, Japan), digitized and analyzed. Immunoreactive cell counting was constantly performed in the dorsal hippocampal region, at the level of the section of 3.80 mm caudal to the bregma, according to Paxinos and Watson stereotaxic atlas. Immunoreactive cell quantification was presented as the mean value of the immunoreactive cells counted in five consecutive hippocampal sections for all investigated regions (CA1, CA2/3, DG) (Supplementary Fig. 1), using Image J software (version $1.52 \mathrm{u}$, U. S. National Institute of Health, Bethesda, Maryland, USA). The counts were made by independent investigators, who were blind to the entire experimental design.

\subsection{Statistical analysis}

SPSS version 20.0 statistical package (IBM SPSS Statistics 20, Chicago, IL, USA) was used for statistical analysis. The results are expressed as mean values \pm standard error of the mean (SEM). After the initial use of the Levene's test for homogeneity of variance and Shapiro-Wilk test of normality, the One-way ANOVA (followed by Bonferroni post hoc test) was used for comparisons between the groups. For all tests, $p<0.05$ was considered significant.

\section{Results \\ 3.1 Anxiety level alterations}

Both behavioral tests performed - OF and EPM test, chosen in accordance with the criteria for an adequate estimation of anxiety. They revealed significant alterations in anxiety state level following the applied protocols (Figs. 1,2). The clear anxiogenic response to chronic intake of nano-sized HA and ACP in OF the significant decrease confirmed the test in cumulative duration and frequency in the center zone ( $\mathrm{df}=$ $6, F=7.033$ and 8.445 , respectively) when compared to the control values (Fig. 1A,B, $p<0.01$ ), with no significant anxiety level alteration after completing the treatment with TCP. However, the anxiogenic response to HA and ACP expressed through the decline of CDCZ was significantly diminished by simultaneous administration of FU extract $(p<0.05)$. However, a significant increase in FCZ after FU administration was observed only in the ACP group $(p<0.01)$. The results obtained in the EPM test (Fig. 2A) showed that only nanosized ACP administration produced a significant decline in cumulative duration in open arms $(\mathrm{F}=9.109, p<0.01)$ that was reversed with FU extract supplementation $(p<0.01)$. As shown in Fig. 2B, all three applied CaPs significantly reduced frequency to open arms $(\mathrm{F}=7.194, p<0.01$ for HA and ACP, and $p<0.05$ for TCP), but this manifestation of anxiogenic response was sufficiently abolished by FU extract only following ACP treatment $(p<0.05)$. The locomotor confirmation of anxiety alteration after completing the treatments with HA, TCP, and ACP, solely and simultaneously with FU 
extract, was estimated using total distance moved and \% of the time moving in both OF and EPM tests (Fig. 1C,D, F $=3.879$ and 1.979; Fig. $2 \mathrm{C}, \mathrm{D}, \mathrm{F}=6.413$ and 5.196, respectively). As an indicator of anxiogenic response, decreased locomotion in OF test was confirmed following HA and ACP nanoparticles administration $(p<0.05)$. In contrast, a significant decline in the same parameter and \% of TM in the EPM test was only observed in the ACP group $(p<0.01)$. Interestingly, the reduction of both TDM and \% of TM in the ACP group was significant even when compared to TCP $(p<0.01)$, but it was successfully eliminated by simultaneous administration of FU extract for both parameters $(p<0.01)$. The anxiogenic response to ACP administration was additionally confirmed by a significant reduction of exploratory activity when expressed as the number of wall rearings in OF and EPM tests (Figs. $1 \mathrm{E}$ and $2 \mathrm{E}, \mathrm{F}=3.108$ and 2.983, respectively) when compared to the control values ( $p<0.05$ and 0.01 , respectively). Even more significant reduction of exploratory activity was observed using the number of free rearings in $\mathrm{OF}$ and EPM tests (Figs. $1 \mathrm{~F}$ and $2 \mathrm{~F}, \mathrm{~F}=8.826$ and 7.610 , respectively). A significant decline of this exploratory indicator was observed in both OF and EPM tests after completing the one-month administration of HA $(p<0.01)$ and ACP $(p<0.05)$, with no significant effect of TCP application. The robust anxiogenic response to ACP was manifested not only when compared to the control values but also in the TCP group $(p<0.05)$.

However, antioxidant supplementation successfully prevented this manifestation of anxiogenic response to HA administration only in an open field. A significant decline in the number of free rearings in elevated plus-maze test after FU application was preserved compared to the control $(p<$ 0.05). The reduction in free exploration for both tests induced by ACP intake was successfully reversed by antioxidant supplementation $(p<0.01)$. Additionally, the possibility for estimation of the exploratory activity in the open arms in the EPM test revealed that the anxiogenic response to CaPs was significant only for unprotected head-dippings (Fig. $2 \mathrm{H}, \mathrm{F}=9.603$ ). At the same time, this was not the case for protected head-dippings (Fig. $2 \mathrm{G}, \mathrm{F}=1.626$ ). The administration of HA and ACP resulted in a significant reduction of this exploratory behavior not only when compared to the control but also when compared to the values to the values observed in the TCP group $(p<0.01)$. At the same time, antioxidant supplementation diminished this manifestation of the anxiogenic response $(p<0.01$ for the ACP + FU group) and returned the values to the control. Ultimately, summarized results for the exploratory activity of both behavioral tests finally showed that protocols with HA and ACP administration produced a significant decline $(p<0.01)$ in the total exploratory activity in OF and EPM test (Figs. 1G and $2 \mathrm{I}, \mathrm{F}=8.686$ and 11.723 , respectively). Not surprisingly, since the EPM test allows a more sensitive insight into exploratory activity, the estimation of the mentioned parameters also showed a significant decline when compared to the
TCP group $(p<0.01)$, but antioxidant supplementation with FU extract at a predefined dose successfully reversed the anxiogenic response to HA and ACP $(p<0.01)$.

\subsection{Impact on oxidative stress markers in hippocampal tissue}

The results presented in Fig. 3 confirmed the strong prooxidant action of the applied protocols. The index of lipid peroxidation, expressed as TBARS (Fig. 3A, F = 9.426), was significantly enhanced by all three nano-sized CaPs intake ( $p$ $<0.01$ for HA and ACP, $p<0.05$ for TCP) when compared to the control. This confirmation of increased ROS production was successfully attenuated by prolonged administration of FU extract, which resulted in a significant decline in the groups of animals treated with ACP $(p<0.01)$. The estimation of antioxidant enzymes activity following the onemonth intake of those mineral components was not uniform. At the same time, the SOD activity (Fig. 3B, F = 2.247) was significantly lowered only in the ACP group ( $p<0.05)$, the diminished antioxidant activity was more pronounced using CAT activity (Fig. 3C, F = 22.818). The pro-oxidative action of nano-sized mineral particles was confirmed by a significant decrease $(p<0.01)$ in CAT activity following administration of all three CaPs. This effect persisted even after simultaneous FU extract application, with the values constantly below the control $(p<0.01)$ except for the TCP + FU group. Interestingly, although antioxidant supplementation along with ACP resulted in a significant increase of CAT activity $(p<0.05)$, but remained lower when compared to control $(p<0.01)$. As shown in Fig. 3D, the administration of those three nano-sized mineral components also significantly affected non-enzymatic antioxidant capacity in the rat hippocampus $(F=6.053)$. Thus, hippocampal GSH levels were significantly lowered $(p<0.01)$ after CaPs application. This effect of the CaPs remained even after simultaneous FU extract administration but was less pronounced in $\mathrm{HA}+\mathrm{FU}$ and $\mathrm{ACP}+\mathrm{FU}$ groups $(p<0.05)$.

\subsection{Alterations in relative pro-and anti-apoptotic gene expression in the hippocampus}

As shown in Fig. 4, the relative gene expression of the pro-apoptotic marker in the hippocampus, estimated using Bax relative gene expression (Fig. 4A), and the hippocampal anti-apoptotic marker - Bcl-2 (Fig. 4B) was significantly altered following the chronic administration of $\mathrm{CaPs}(\mathrm{df}=6$, $\mathrm{F}=3.896$ and 23.364, respectively). The increase in rat hippocampal Bax relative gene expression following prolonged ACP application was evident compared to control values $(p<$ 0.05 ), but the other mineral composites produced no significant effect. Alternatively, the pro-apoptotic influence of ACP was successfully abolished by FU extract administration $(p<$ 0.05 ). At the same time, the impact of individual nano-sized CaPs on the anti-apoptotic capacity of rat hippocampal tissue was even clearer. Namely, all three applied mineral components significantly reduced $\mathrm{Bcl}-2$ relative gene expression after one month of treatment $(p<0.01)$. The decline in Bcl-2 gene expression was neutralized by chronic FU extract intake 

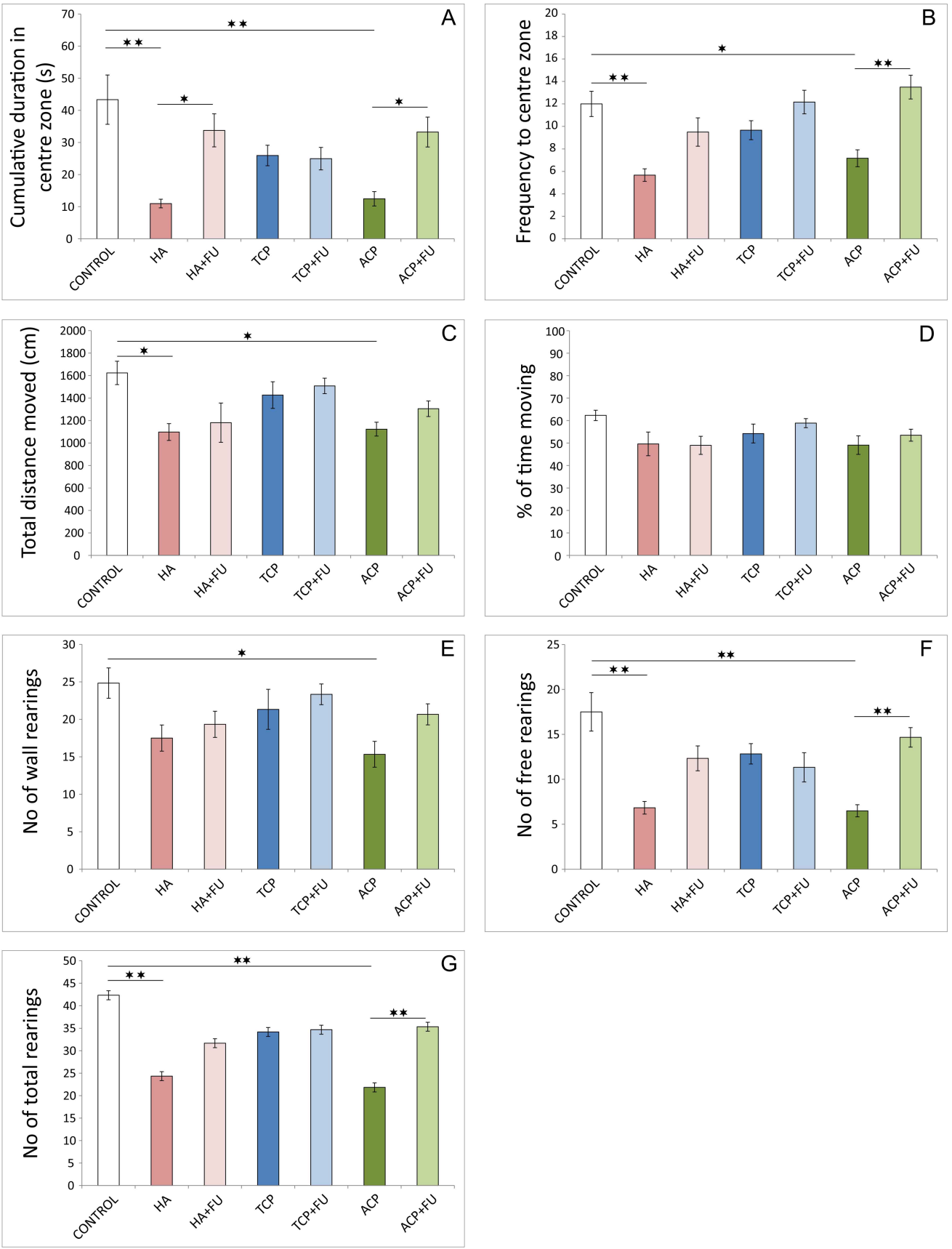

Fig. 1. Anxiety indicators were obtained in the open field test (6 per group). (A) Cumulative duration in the center zone. (B) Frequency to center zone. (C) Total distance moved. (D) Percentage of time moving. (E) The number of wall rearings. (F) The number of free rearings. (G) The number of total rearings. HA - hydroxyapatite; HA + FU - hydroxyapatite and Filipendula ulmaria extract; TCP - tricalcium phosphate hydrate; TCP + FU - tricalcium phosphate hydrate and Filipendula ulmaria extract; ACP - amorphous calcium phosphate; ACP + FU - amorphous calcium phosphate and Filipendula ulmaria extract. The graph bars present mean $\pm \mathrm{SEM},{ }^{*}$ represents a significant difference $p<0.05$, ${ }^{* *}$ represents a significant difference $p<0.01$.

in all three groups $(p<0.01)$, reversing the hippocampal Bcl2 relative gene expression to control values. As the mineral components had more effect on anti-apoptotic activity than on pro-apoptotic marker (Bax) expression, it is not surprising that the $\mathrm{Bax} / \mathrm{Bcl}-2$ ratio (Fig. $4 \mathrm{C}, \mathrm{F}=35.992$ ) mostly resembles the results obtained for $\mathrm{Bcl}-2$.
3.4 Immunohistochemical verification of BDNF and GABA-AR2S changes in hippocampal tissue

Immunohistochemical analysis also revealed a significant impact of the pretreatment protocols on the number of brainderived neurotrophic factor (BDNF) (Fig. 5) and GABAAR2S (Fig. 6) immunoreactive cells in the rat hippocampus. The total number of BDNF immunoreactive cells in 

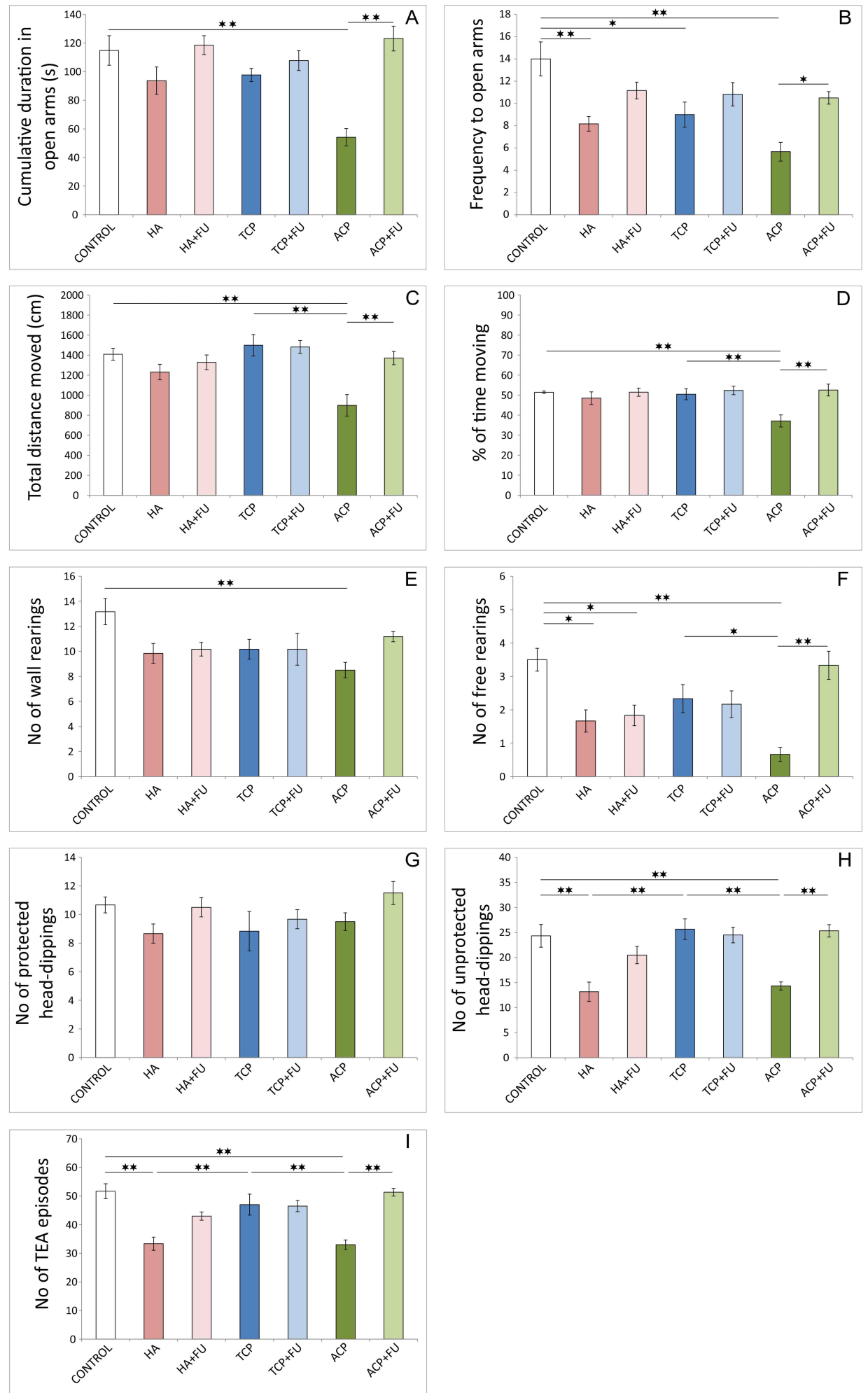

Fig. 2. Anxiety indicators were obtained in the elevated plus-maze test (6 per group). (A) Cumulative duration in open arms. (B) Frequency to open arms. (C) Total distance moved. (D) Percentage of time moving. (E) The number of free rearings. (F) The number of wall rearings. (G) The number of protected head-dippings. (H) The number of unprotected head-dippings. (I) The number of TEA (total exploratory activity) episodes. HA - hydroxyapatite; HA + FU - hydroxyapatite and Filipendula ulmaria extract; TCP - tricalcium phosphate hydrate; TCP + FU - tricalcium phosphate hydrate and Filipendula ulmaria extract; ACP - amorphous calcium phosphate; ACP + FU - amorphous calcium phosphate and Filipendula ulmaria extract. The graph bars present mean \pm SEM, * represents a significant difference $p<0.05$, ${ }^{* *}$ represents a significant difference $p<0.01$. 


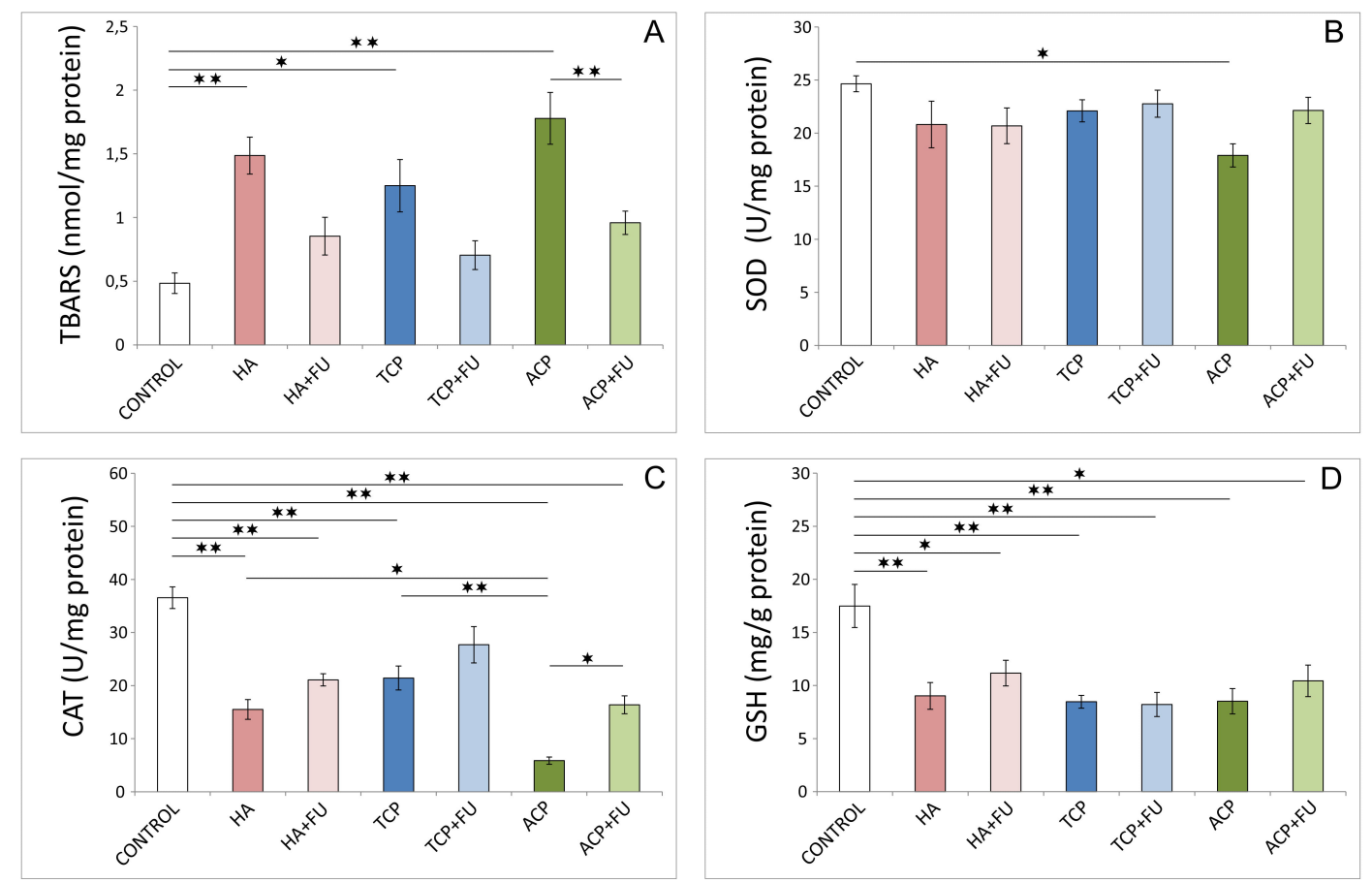

Fig. 3. Hippocampal oxidative stress markers (6 per group). (A) Index of lipid peroxidation (expressed as thiobarbituric acid reactive substances - TBARS). (B) CAT (catalase) activity. (C) SOD (superoxide dismutase) activity. (D) GSH (reduced glutathione). HA - hydroxyapatite; HA + FU - hydroxyapatite and Filipendula ulmaria extract; TCP - tricalcium phosphate hydrate; TCP + FU - tricalcium phosphate hydrate and Filipendula ulmaria extract; ACP - amorphous calcium phosphate; ACP + FU - amorphous calcium phosphate and Filipendula ulmaria extract. The graph bars present mean \pm SEM, * represents a significant difference $p<0.05{ }^{* *}$ represents a significant difference $p<0.01$.

the entire hippocampal sections (Fig. 5D) was significantly reduced by the one-month administration of all $\mathrm{CaPs}$ ( $\mathrm{F}$ $=79.621, p<0.01)$. Simultaneous application of FU extract did not prevent the observed reduction in BDNF immunoreactive cells following prolonged intake of HA, TCP, and ACP, which remained at levels beyond the control values $(p<0.01)$. However, the FU extract significantly increased BDNF immunoreactive cells number when compared to the groups where HA $(p<0.01)$ and ACP $(p<0.05)$ were applied separately. Interestingly, in those groups, the hippocampal BDNF immunoreactivity was significantly lower even when compared to the TCP group $(p<0.01)$. Analysis of individual hippocampal regions showed that the strongest impact $(F=88.388)$ of the applied protocols was observed in DG (Fig. 5C), where all mineral components applications significantly reduced the number of BDNF immunoreactive cells $(p<0.01)$. The reduction achieved in the HA group was also significant compared to the TCP group $(p<0.01)$. Although the administration of FU extract significantly increased BDNF immunoreactivity in DG when applied along with HA $(p<0.01)$, the number of BDNF immunoreactive cells in all combined groups was still significantly lower when compared to control values $(p<0.01)$. There was a less pronounced response $(\mathrm{F}=13.575)$ to the applied nanosized mineral components using the number of BDNF immunoreactive cells in the CA2/3 region (Fig. 5B) since only
HA and ACP groups showed a significant decline in BDNF immunoreactivity when compared to the control but also the TCP group $(p<0.01)$. The protective role of FU extract on BDNF immunoreactivity in the $\mathrm{CA} 2 / 3$ region was confirmed by preventing a significant decline in the number of BDNF immunoreactive cells in $\mathrm{HA}+\mathrm{FU}$ and $\mathrm{ACP}+\mathrm{FU}$ groups when compared to the control values. Only the slightest effect $(F=5.396)$ of the applied mineral components was observed in the CA1 region (Fig. 5A). In contrast, the number of BDNF immunoreactive cells was reduced in the CA1 region by HA and TCP application $(p<0.05)$, while the effect of the treatment with ACP was even more pronounced ( $p<$ 0.01 ).

As shown in Fig. 6, the applied protocols affected the number of GABA-AR2S immunoreactive cells in CA1, CA2/3, DG, and total hippocampal sections ( $F=19.839$, $13.006,10.745$, and 32.343, respectively). HA and ACP application resulted in a significant decline of the number of GABA-AR2S immunoreactive cells in all investigated regions (Fig. 6A-C), as well as in total hippocampal GABAAR2S immunoreactivity (Fig. 6D). The same effect was observed in the TCP group, but the impact of TCP administration was slightly lower in the CA1 region $(p<0.05)$ when compared to the control. Likewise, FU extract and nanosized mineral components administration prevented any significant decline in the number of GABA-AR2S immunore- 

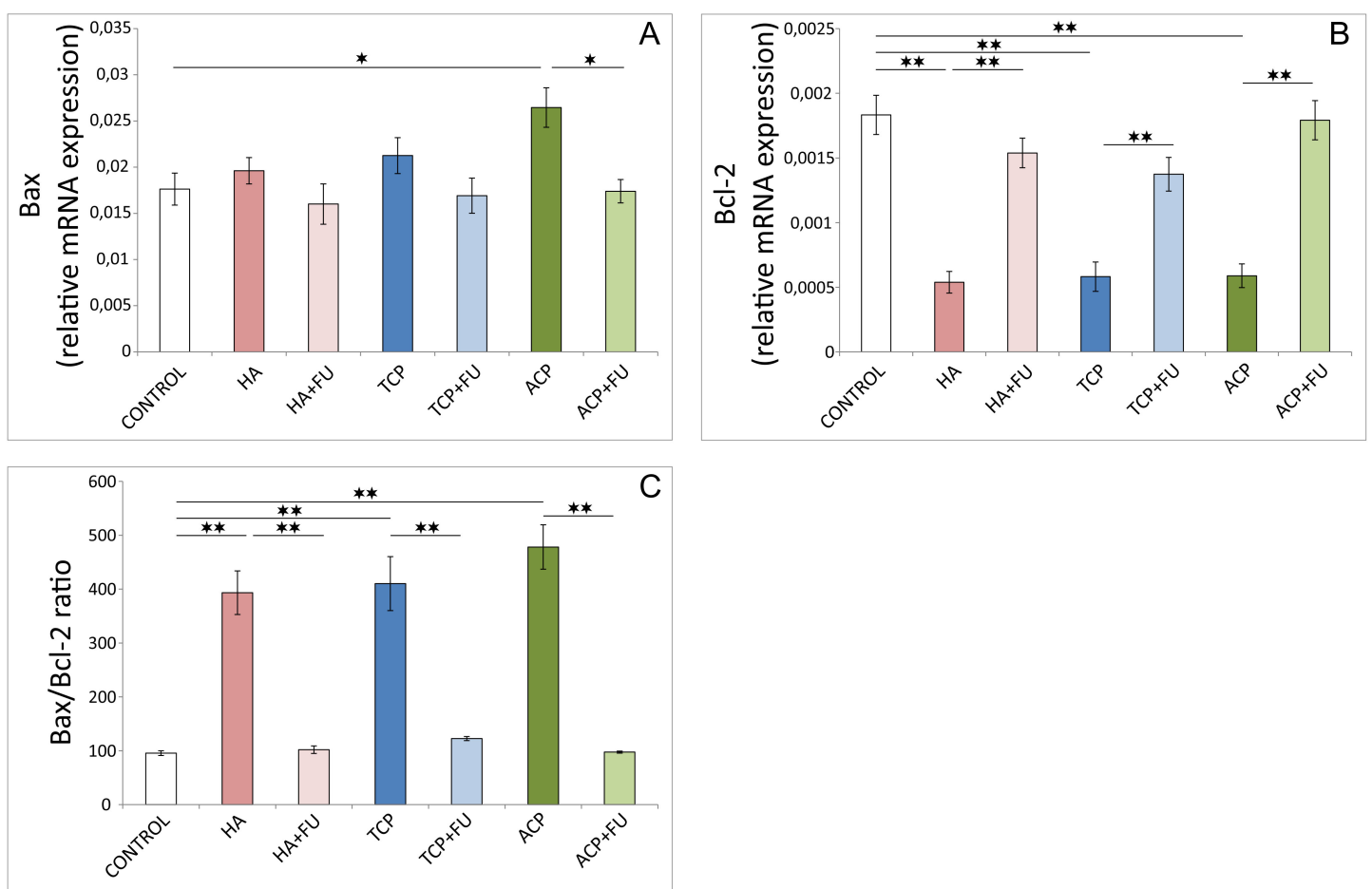

Fig. 4. Pro-and anti-apoptotic relative gene expression in the hippocampus (6 per group). (A) Bax. (B) Bcl-2. (C) Bax/Bcl-2 ratio. HA - hydroxyapatite; $\mathrm{HA}+\mathrm{FU}$ - hydroxyapatite and Filipendula ulmaria extract; TCP - tricalcium phosphate hydrate; TCP + FU - tricalcium phosphate hydrate and Filipendula ulmaria extract; ACP - amorphous calcium phosphate; ACP + FU - amorphous calcium phosphate and Filipendula ulmaria extract. The graph bars present mean $\pm \mathrm{SEM}$, * represents a significant difference $p<0.05$, ** represents a significant difference $p<0.01$.

active cells only when applied simultaneously with TCP in the CA1 region. The results observed in other combined groups and TCP + FU in other regions confirmed that the applied antioxidant supplementation was insufficient to diminish the decline in hippocampal GABA-AR2S immunoreactivity since the number of GABA-AR2S immunoreactive cells remained below the control values.

\section{Discussion}

The widespread medical use of nanoparticles, including $\mathrm{CaPs}$, is associated with confirmed toxicities in numerous organs and tissues. Further elucidation is therefore required to determine the general mechanism (including oxidative damage) that may compromise their efficiency and safety. Consequently, it seems appropriate to evaluate and promote the prevention of nanoparticle-induced toxicities by antioxidant supplementation. Furthermore, there is a growing need for antioxidant components obtained from natural products. When taken together, this allows the two current medical approaches explored here (the employment of nanomaterials and simultaneous attenuation of their toxicities) to complement each other.

As described above, it is clear that the CaP compounds applied in nanoparticles significantly altered numerous parameters estimated. This is not surprising since literature data indicate different pathways for the entrance of nanopar- ticles of other substances into the central nervous system, accompanied by neurotoxicity with behavioral manifestations [21]. Also, there is evidence that nano-CaP compounds enter numerous brain regions, including the hippocampus [22], which is the initiating point for this investigation, confirming earlier research that concluded that these compounds induce neurotoxicity with a prodepressant outcome and cognitive impairment [10].

\subsection{Pro-oxidant and pro-apoptotic action of nano-CaPs}

The impact of $\mathrm{CaP}$ nanoparticles employed on oxidative stress markers (Fig. 3) is in accordance with the results of previous investigations that analyzed the mechanisms of those nanoparticles' toxicities. As a postulated mechanism, it has been suggested that nano-HA increased ROS generation, predominantly via hydroxyl radical production [23]. Furthermore, the oxidative imbalance induced by nano-HA has been associated with a decline in cellular antioxidant capacity expressed as a reduction in SOD activity in C6 cell cultures [1] Results obtained confirmed that antioxidant enzyme activity was more affected by the diminished CAT activity. Interestingly, the values that resume the cellular antioxidant capacity based on antioxidant enzyme activity were not uniformly improved by antioxidant supplementation, while the nonenzymatic antioxidant defense system depletion (expressed by GSH) was not successfully recovered by FU extract. 

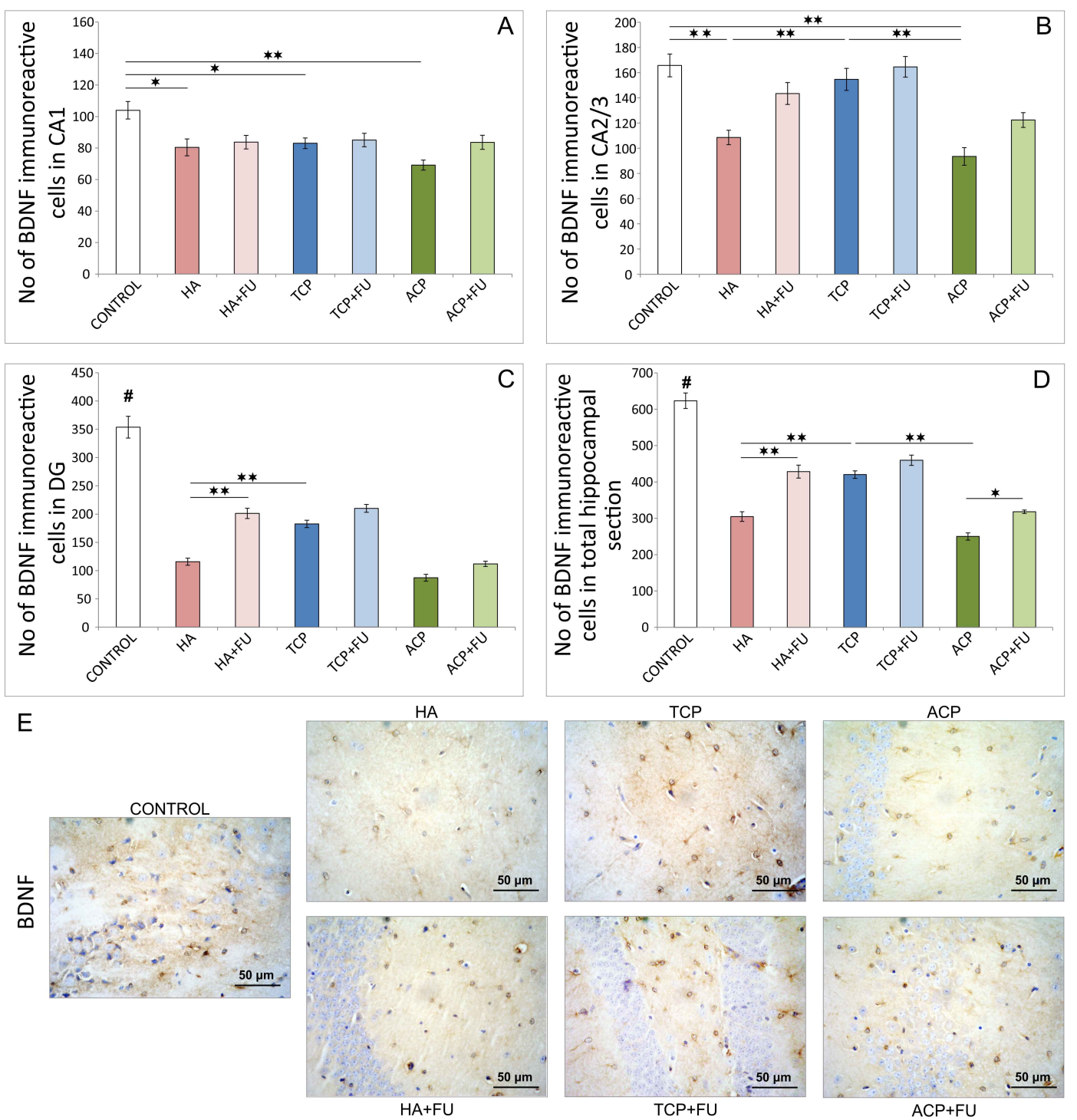

Fig. 5. Quantitative immunohistochemical analysis of BDNF-positive cells in the hippocampus (6 per group) and the distribution in CA1 region (A), CA2/3 region (B), DG (C), and the total section of the hippocampus (D), and (E) the representative images of BDNF IHC staining (original magnification $\times 40$ ). HA - hydroxyapatite; HA + FU - hydroxyapatite and Filipendula ulmaria extract; TCP - tricalcium phosphate hydrate; TCP + FU - tricalcium phosphate hydrate and Filipendula ulmaria extract; ACP - amorphous calcium phosphate; ACP + FU - amorphous calcium phosphate and Filipendula ulmaria extract. The graph bars present mean \pm SEM, ${ }^{*}$ represents a significant difference $p<0.05,{ }^{* *}$ represents a significant difference $p<0.01$, \# represents a significant difference $(p<0.01)$ between control and all other groups.

There is considerable evidence that oxidative damage significantly affects the balance between pro-and anti-apoptotic factors [24]. Following the results for oxidative stress markers obtained, it is not surprising that the applied protocols with nano-sized CaPs potentiated the pro-apoptotic outcome (Fig. 4). Namely, the direct impact of mineral components on the augmentation of pro-apoptotic relative gene expression (Bax) was significant only in the ACP group. The minimization of anti-apoptotic relative gene expression (Bcl-2) was observed for all three mineral components administered. Antioxidant supplementation with FU extract was beneficial using attenuation of pro-apoptotic and potentiating anti- apoptotic relative gene expression. This was confirmed by improving the $\mathrm{Bax} / \mathrm{Bcl}-2$ ratio observed following prolonged administration of mineral components.

The results obtained are concordant with previous reports that evaluated the mechanisms of $\mathrm{CaP}$-induced apoptosis. Principally, it seems that both the size and the chemical structure of mineral components significantly affect apoptotic mechanisms in various tissues. In general, metallic nanoparticles have been reported to increase NO release and proinflammatory factors such as tumor necrosis factor-alpha by activated microglia in cell culture [25]. Considering the impact of chemical structure on the apoptotic response to CaPs, 

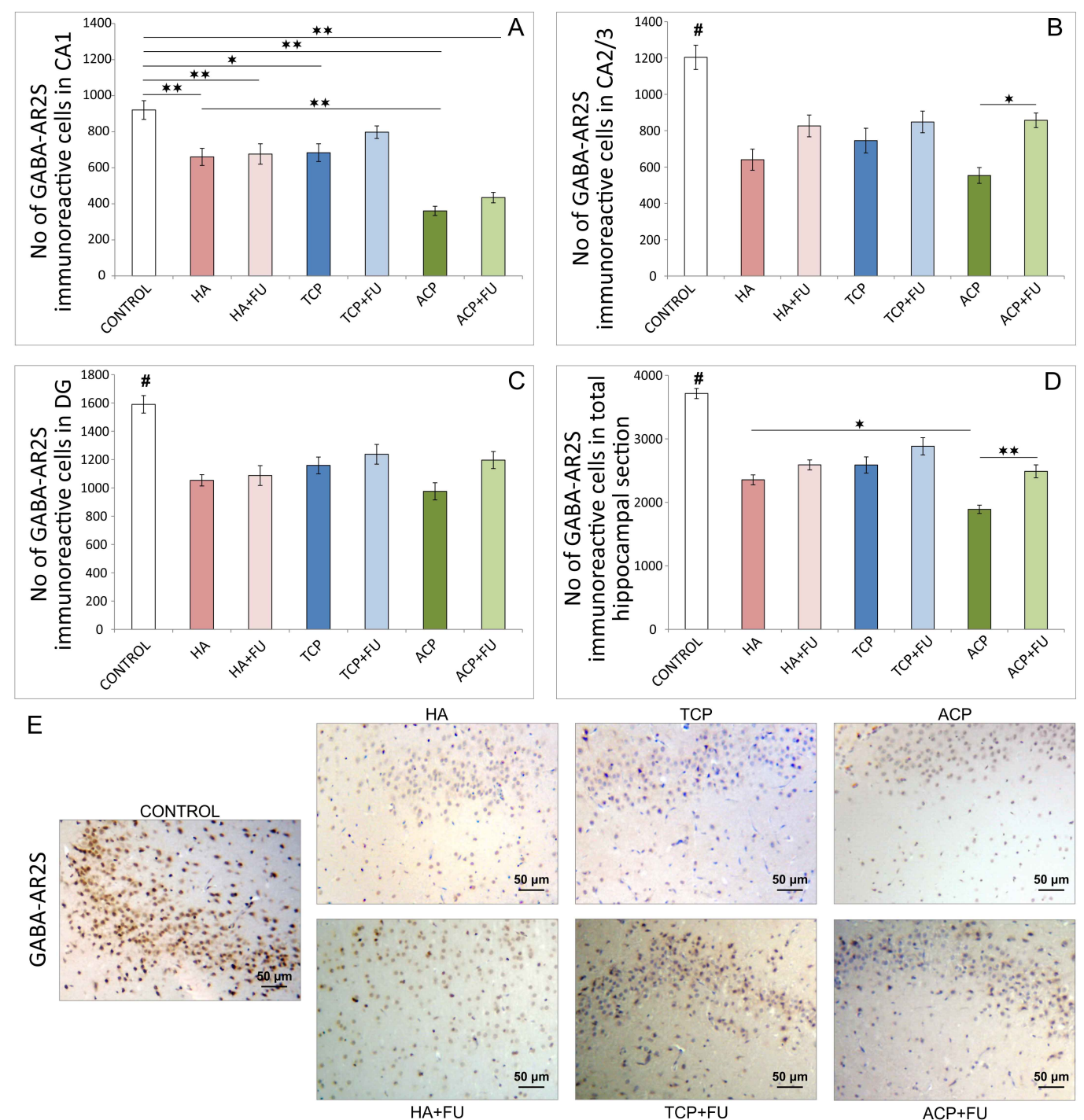

Fig. 6. Quantitative immunohistochemical analysis of GABA-AR2S-positive cells in the hippocampus (6 per group) and the distribution in CA1 region (A), CA2/3 region (B), DG (C), and the total section of the hippocampus (D), and (E) the representative images of GABA-AR2S IHC staining (original magnification x20). HA - hydroxyapatite; HA + FU - hydroxyapatite and Filipendula ulmaria extract; TCP - tricalcium phosphate hydrate; TCP + FU - tricalcium phosphate hydrate and Filipendula ulmaria extract; ACP - amorphous calcium phosphate; ACP + FU - amorphous calcium phosphate and Filipendula ulmaria extract. The graph bars present mean $\pm \mathrm{SEM},{ }^{*}$ represents a significant difference $p<0.05$, ${ }^{* *}$ represents a significant difference $p<0.01$, \# represents a significant difference $(p<0.01)$ between control and all other groups.

it should be mentioned that HA nanoparticle administration was associated with an increased p53, down-regulation of Bcl-2, and general DNA damage in rat kidneys [26]. At the same time, nano-ACP can cause apoptosis of the leukemia P388 cells by selectively acting on G1 in the cell cycle [27]. Increased intracellular calcium level (probably due to its release from nanoparticles) has been reported to trigger apoptosis [28]. Additionally, increased intracellular PO43- has been suggested to induce apoptosis through modulation of the mitochondrial membrane potential [29]. Generally, it should be noted that there is still no consensus as to whether CaPs induce apoptosis via caspase-dependent or independent pathways [30].

\subsection{Down-regulation of hippocampal BDNF and GABA-AR following nano-CaPs intake}

The pro-apoptotic action of all three mineral components applied accompanied hippocampal BDNF decline (Fig. 5). Although it is well known that BDNF has anti-apoptotic potential in various tissues [31], including CNS [32], it has also been reported that apoptosis itself, especially in the regions responsible for BDNF production, may significantly reduce neurogenesis [33], thus affecting BDNF content. Indeed, the results confirmed that the groups with mineral components intake, with a marked pro-apoptotic outcome, also expressed the lowest hippocampal BDNF immunoreactivity. Furthermore, since BDNF is highly responsible 
for hippocampal neurogenesis [34], it is not surprising that GABA-AR were also at their lowest levels in the groups (HA and ACP) with evident pro-apoptotic action and downregulation of hippocampal BDNF (Fig. 6). Our results also agree with a confirmed connection between increased BDNF and up-regulation of the GABAergic system [35].

\subsection{Anxiogenic response to nano-CaPs}

Finally, the results of behavioral testing performed strongly confirmed an anxiogenic response to the administered mineral components (Figs. 1,2), with the most pronounced effect observed with HA, especially ACP nanoparticles. Increased anxiety following prolonged HA and ACP application may be associated with the previously discussed down-regulation of the hippocampal GABAergic system, expressed using a decline in the number of GABA-AR2S immunoreactive cells in all investigated hippocampal regions. The results obtained for the alterations in anxiety level that correspond to hippocampal GABA-AR expression follow previously reported evidence that GABAergic dysfunction plays one of the key roles in the pathogenesis of various mood disorders [36] and anxiety [37, 38]. Moreover, it should be mentioned that considerable evidence was found for the impact of some of the other parameters evaluated contributing to anxiety level regulation. Therefore, it has been reported that the experimental protocols that resulted in pro-oxidative [39] and pro-apoptotic [13] action in hippocampal tissue significantly enhanced anxiogenic response. At the same time, the down-regulation of hippocampal BDNF was also associated with increased anxiety [40]. Although not evaluated in this study, the additional pathophysiological mechanism for mood alterations could be found in astrocytic impairment in rat hippocampus that affects other transmitters and result in mood deprivation [41].

\subsection{Protective role of FU extract on anxiogenic response to nano-CaPs}

The results considering the consequences of this antioxidant-rich FU extract usage confirmed that this therapeutic approach might be beneficial in the prevention of the described mood alterations induced by nano-CaPs. At the dose applied, the potentially protective role of FU extract was confirmed by its attenuation of numerous anxiogenic factors such as oxidative damage, up-regulation of antiapoptotic and down-regulation pro-apoptotic factors well as increased hippocampal BDNF and GABA-AR2S expression. The improvement in anxiety level regulation parameters following antioxidant supplementation observed further confirms the findings of recently published investigations performed in our lab [40].

\section{Conclusions}

This research analyzed the possible influence of an antioxidant-rich extract of FU on anxiety level alterations induced by nano-sized particles of different CaPs. As previously noted, this investigation, based on the results obtained in pre- vious work [42], should be followed by a broader approach that includes other nano-CaPs, with a different experimental design (including more prolonged treatment, and finally should explore other brain regions responsible for mood regulation).

The results obtained suggest a growing need for new materials in restorative dentistry should include, besides the estimation of local tissue response, an adequate methodology for the necessary estimation of various potential side effects, including neurotoxicity and its behavioral manifestations. It is worth noting that some differences for individual nanoCaPs were observed for the behavioral manifestations of neurotoxicity. From this perspective, it would appear that TCP was less harmful using anxiety level alteration following the protocols applied herein. Additionally, it seems that antioxidant supplementation may be useful in the prevention and/or treatment of toxicities induced by CaPs.

\section{Abbreviations}

$\mathrm{CaP}$, calcium phosphate; HA, hydroxyapatite; TCP, tricalcium phosphate; ACP, amorphous calcium phosphate; FU, Filipendula ulmaria; OF, open field; EPM, elevated plus maze; $\mathrm{CDCZ}$, cumulative duration in the center zone; FCZ, frequency to the central zone; TDM, total distance moved; \%TM, percentage of time moving; CDOA, cumulative duration in open arms; FOA, frequency to open arms; TEA, total exploratory activity; SOD, superoxide dismutase; CAT, catalase; GSH, glutathione.

\section{Author contributions}

Conceptualization-NA, DS, NJ and GR; methodologyNA, DS, NJ and GR; formal analysis and investigationNA, DS, JSKS, VM, SM, JM, PM, MV, AN, OM-DJ, MZ, $\mathrm{NF}, \mathrm{VJ}, \mathrm{NJ}$ and GR; resources-NA, DS, VJ, NJ and GR; data curation-NA, DS, NJ and GR; writing-original draft preparation-NA, DS, NJ and GR; writing-review and editing-NA, DS, NJ and GR; visualization-NA, DS, NJ and GR. All authors have read and agreed to the published version of the manuscript.

\section{Ethics approval and consent to participate}

The pre-treatment and experimental procedures were performed following the European Directive for the Welfare of Laboratory Animals Directive 2010/63/EU (ethic code: 01-1377/2021), and the GLP principles, as well as the ARRIVE guidelines, following the approval by the Ethical Committee of the Faculty of Medical Sciences, University of Kragujevac, Serbia.

\section{Acknowledgment}

This work was supported by the Faculty of Medical Sciences, University of Kragujevac, Serbia (JP 01/19), and Ministry of Education, Science, and Technological Development of the Republic of Serbia 451-03-68/2020-14/200107 (Faculty of Engineering, University of Kragujevac), 451-03- 
68/2020-14/200378 (Institute for Information Technologies Kragujevac, University of Kragujevac) and 451-03-68/202014/200122 (Faculty of Science).

\section{Funding}

This research received no external funding.

\section{Conflict of interest}

The authors declare no conflict of interest.

\section{Supplementary material}

Supplementary material associated with this article can be found, in the online version, at https://www.imrpress.com/j ournal/JIN/20/4/10.31083/j.jin2004089.

\section{References}

[1] Xu J, Xu P, Li Z, Huang J, Yang Z. Oxidative stress and apoptosis induced by hydroxyapatite nanoparticles in C6 cells. Journal of Biomedical Materials Research. Part A. 2012; 100: 738-745.

[2] Xu Z, Liu C, Wei J, Sun J. Effects of four types of hydroxyapatite nanoparticles with different nanocrystal morphologies and sizes on apoptosis in rat osteoblasts. Journal of Applied Toxicology. 2012; 32: 429-435.

[3] Shi Z, Huang X, Cai Y, Tang R, Yang D. Size effect of hydroxyapatite nanoparticles on proliferation and apoptosis of osteoblastlike cells. Acta Biomaterialia. 2009; 5: 338-345.

[4] Liu H, Yazici H, Ergun C, Webster TJ, Bermek H. An in vitro evaluation of the $\mathrm{Ca} / \mathrm{P}$ ratio for the cytocompatibility of nanoto-micron particulate calcium phosphates for bone regeneration. Acta Biomaterialia. 2008; 4: 1472-1479.

[5] Barros L, Cabrita L, Boas MV, Carvalho AM, Ferreira ICFR. Chemical, biochemical and electrochemical assays to evaluate phytochemicals and antioxidant activity of wild plants. Food Chemistry. 2011; 127: 1600-1608.

[6] Katanić J, Boroja T, Stanković N, Mihailović V, Mladenović M, Kreft S, et al. Bioactivity, stability and phenolic characterization of Filipendula ulmaria (L.) Maxim. Food \& Function. 2015; 6: 11641175.

[7] Katanić J, Matić S, Pferschy-Wenzig E, Kretschmer N, Boroja $\mathrm{T}$, Mihailović $\mathrm{V}$, et al. Filipendula ulmaria extracts attenuate cisplatin-induced liver and kidney oxidative stress in rats: in vivo investigation and LC-MS analysis. Food and Chemical Toxicology. 2017; 99: 86-102.

[8] Proestos C, Zoumpoulakis P, Sinanoglou VJ. Determination of Plant Bioactive Compounds. Antioxidant Capacity and Antimicrobial Screening. Focusing on Modern Food Industry. 2013; 2: 26-35.

[9] Yu L, Tao J, Zhao Q, Xu C, Zhang Q. Confirmation of potential neuroprotective effects of natural bioactive compounds from traditional medicinal herbs in cerebral ischemia treatment. Journal of Integrative Neuroscience. 2020; 19: 373-384.

[10] Arsenijevic N, Selakovic D, Katanic Stankovic JS, Mihailovic V, Mitrovic S, Milenkovic J, et al. The Beneficial Role of Filipendula ulmaria Extract in Prevention of Prodepressant Effect and Cognitive Impairment Induced by Nanoparticles of Calcium Phosphates in Rats. Oxidative Medicine and Cellular Longevity. 2021; 2021: 6670135.

[11] Wang L, Zhou G, Liu H, Niu X, Han J, Zheng L, et al. Nanohydroxyapatite particles induce apoptosis on MC3T3-E1 cells and tissue cells in SD rats. Nanoscale. 2012; 4: 2894-2899.

[12] Zhang L, Weir MD, Chow LC, Antonucci JM, Chen J, Xu HHK. Novel rechargeable calcium phosphate dental nanocomposite. Dental Materials. 2016; 32: 285-293.
[13] Selakovic D, Joksimovic J, Zaletel I, Puskas N, Matovic M, Rosic G. The opposite effects of nandrolone decanoate and exercise on anxiety levels in rats may involve alterations in hippocampal parvalbumin-positive interneurons. PLoS ONE. 2017; 12: e0189595.

[14] Kumburovic I, Selakovic D, Juric T, Jovicic N, Mihailovic V, Stankovic JK, et al. Antioxidant Effects of Satureja hortensisL. Attenuate the Anxiogenic Effect of Cisplatin in Rats. Oxidative Medicine and Cellular Longevity. 2019; 2019: 8307196.

[15] Lowry OH, Rosebrough NJ, Farr AL, Randall RJ. Protein measurement with the Folin phenol reagent. Journal of Biological Chemistry. 1951; 193: 265-275.

[16] Ohkawa H, Ohishi N, Yagi K. Assay for lipid peroxides in animal tissues by thiobarbituric acid reaction. Analytical Biochemistry. 1979; 95: 351-358.

[17] Misra HP, Fridovich I. The role of superoxide anion in the autoxidation of epinephrine and a simple assay for superoxide dismutase. The Journal of Biological Chemistry. 1972; 247: 3170-3175.

[18] Beers RF, Sizer IW. A spectrophotometric method for measuring the breakdown of hydrogen peroxide by catalase. The Journal of Biological Chemistry. 1952; 195: 133-140.

[19] Ellman GL. Tissue sulfhydryl groups. Archives of Biochemistry and Biophysics. 1959; 82: 70-77.

[20] Livak KJ, Schmittgen TD. Analysis of relative gene expression data using real-time quantitative PCR and the 2 (-Delta Delta $\mathrm{C}(\mathrm{T})$ ) Method. Methods. 2001; 25: 402-408.

[21] Feng X, Chen A, Zhang Y, Wang J, Shao L, Wei L. Application of dental nanomaterials: potential toxicity to the central nervous system. International Journal of Nanomedicine. 2015; 10: $3547-$ 3565.

[22] Abbas OA, Ibrahim IG, Ismail AE. Therapeutic Effects of NanoHAp in a Rat Model of AlCl3 Induced Neurotoxicity. Iranian Journal of Pharmaceutical Research. 2019; 18: 1309-1322.

[23] Liu R, Narla RK, Kurinov I, Li B, Uckun FM. Increased hydroxyl radical production and apoptosis in PC12 neuron cells expressing the gain-of-function mutant G93a SOD1 gene. Radiation Research. 1999; 151: 133-141.

[24] Redza-Dutordoir M, Averill-Bates DA. Activation of apoptosis signaling pathways by reactive oxygen species. Biochimica et Biophysica Acta. 2016; 1863: 2977-2992.

[25] Xue Y, Wu J, Sun J. Four types of inorganic nanoparticles stimulate the inflammatory reaction in brain microglia and damage neurons in vitro. Toxicology Letters. 2012; 214: 91-98.

[26] Mosa IF, Youssef M, Kamel M, Mosa OF, Helmy Y. Synergistic antioxidant capacity of CsNPs and CurNPs against cytotoxicity, genotoxicity and pro-inflammatory mediators induced by hydroxyapatite nanoparticles in male rats. Toxicology Research. 2019; 8: 939-952.

[27] Li G, Huang J, Li Y, Zhang R, Deng B, Zhang J, et al. In vitro study on influence of a discrete nano-hydroxyapatite on leukemia P388 cell behavior. Bio-Medical Materials and Engineering. 2007; 17: 321-327.

[28] Masouleh MP, Hosseini V, Pourhaghgouy M, Bakht MK. Calcium Phosphate Nanoparticles Cytocompatibility Versus Cytotoxicity: A Serendipitous Paradox. Current Pharmaceutical Design. 2017; 23: $2930-2951$

[29] Miedlich SU, Zalutskaya A, Zhu ED, Demay MB. Phosphateinduced apoptosis of hypertrophic chondrocytes is associated with a decrease in mitochondrial membrane potential and is dependent upon Erk1/2 phosphorylation. The Journal of Biological Chemistry. 2010; 285: 18270-18275.

[30] Meena R, Kesari KK, Rani M, Paulraj R. Effects of hydroxyapatite nanoparticles on proliferation and apoptosis of human breast cancer cells (MCF-7). Journal of Nanoparticle Research. 2012; 14: 111.

[31] Tezcan B, Hacıoglu G, Abidin S, Abidin İ. Apoptotic Effects of Reduced Brain-Derived Neurotrophic Factor (BDNF) on Mouse Liver and Kidney. Dicle Tıp Dergisi. 2017; 44: 315-324. 
[32] Xu D, Lian D, Wu J, Liu Y, Zhu M, Sun J, et al. Brain-derived neurotrophic factor reduces inflammation and hippocampal apoptosis in experimental Streptococcus pneumoniae meningitis. Journal of Neuroinflammation. 2017; 14: 156.

[33] Ryu JR, Hong CJ, Kim JY, Kim E, Sun W, Yu S. Control of adult neurogenesis by programmed cell death in the mammalian brain. Molecular Brain. 2016; 9: 43.

[34] Scharfman H, Goodman J, Macleod A, Phani S, Antonelli C, Croll $\mathrm{S}$. Increased neurogenesis and the ectopic granule cells after intrahippocampal BDNF infusion in adult rats. Experimental Neurology. 2005; 192: 348-356.

[35] Pillai A. Brain-derived neurotrophic factor/TrkB signaling in the pathogenesis and novel pharmacotherapy of schizophrenia. Neurosignals. 2008; 16: 183-193.

[36] Brambilla P, Perez J, Barale F, Schettini G, Soares JC. GABAergic dysfunction in mood disorders. Molecular Psychiatry. 2003; 8: 721-715.

[37] Brambilla P, Barale F, Caverzasi E, Soares JC. Anatomical MRI findings in mood and anxiety disorders. Epidemiologia E Psichiatria Sociale. 2002; 11: 88-99.
[38] Naseri M, Hesami-Tackallou S, Torabi-Nami M, Zarrindast M, Nasehi M. Involvement of the CA1 GABAA receptors in MK801-induced anxiolytic-like effects: an isobologram analysis. Behavioural Pharmacology. 2014; 25: 197-205.

[39] Vukovic R, Kumburovic I, Joksimovic Jovic J, Jovicic N, Katanic Stankovic JS, Mihailovic V, et al. N-Acetylcysteine Protects against the Anxiogenic Response to Cisplatin in Rats. Biomolecules. 2019; 9: 892 .

[40] Stajic D, Selakovic D, Jovicic N, Joksimovic J, Arsenijevic N, Lukic $\mathrm{ML}$, et al. The role of galectin-3 in modulation of anxiety state level in mice. Brain, Behavior, and Immunity. 2019; 78: 177-187.

[41] Jiang S, Zhang Q, Guo Q, Di Z. The glutamatergic system and astrocytic impairment in rat hippocampus: a comparative study of underlying etiology and pathophysiology of depression. Journal of Integrative Neuroscience, 2019; 18: 387-392.

[42] Parayanthala Valappil M, Santhakumar S, Arumugam S. Determination of oxidative stress related toxicity on repeated dermal exposure of hydroxyapatite nanoparticles in rats. International Journal of Biomaterials. 2014; 2014: 476942. 